\title{
Quantitative Measurement and Utilization of Electron Irradiation Effects in 2D Materials
}

Gregor Leuthner, Thuy An Bui, Georg Zagler, Bernhard Fickl, Mohammad Monazam, Alexandru Chirita, Toma Susi and Jani Kotakoski

University of Vienna, Vienna, Wien, Austria

As is clear for every electron microscopist, electrons are strongly interacting particles and when traversing a material often cause changes in its atomic structure.

The electron-sample interactions can be divided into elastic and inelastic scattering events, where the first ones lead to excitations with an associated loss of electron energy, and the second ones to momentum transfer from the electron to a sample nucleus. Depending on the electronic structure of the sample material as well as the electron energy either one of these scattering mechanisms can be dominant, or they can both contribute to changes in the sample. In addition, chemical effects are also possible due to surface contamination on the sample or less-than-ideal vacuum in the objective area of the microscope.

All of these effects are well known, and have been studied for decades. However, only the combination of aberration-corrected instruments and two-dimensional materials have made it truly possible to study them quantitatively [1]. Until now, only knock-on damage in graphene, resulting from elastic scattering, has been satisfactorily explained through theory [2,3] while quantitative measurements for semiconducting or insulating materials where inelastic scattering becomes important have been lacking except for few exceptions [4]. Chemical effects have received even less attention due to the lack of suitable instrumentation.

In this contribution, we will overview the latest quantitative results on irradiation effects in hexagonal boron nitride for energies between 55 and $100 \mathrm{keV}$, chemical effects on atomic structure of graphene, $\mathrm{MoS}_{2}$ and $\mathrm{MoTe}_{2}$ in the electron microscope under low-pressure oxygen atmospheres, and electron-beamdriven 2D to 3D structural transformation in a novel 2D CuAu structure [5], among other recent studies.

These results contribute to the growing literature on atomic-scale structural engineering in the electron microscope bringing us ever closer to tailor-made atomic structures with unique properties for future applications.

\section{References}

[1] Susi, Meyer, Kotakoski, Nat. Rev. Phys. 1, 397 (2019)

[2] Meyer et al., Phys. Rev. Lett. 108, 196102 (2012)

[3] Susi et al., Nat. Commun. 7, 13040 (2016)

[4] Cretu, Lin, Suenaga, Micron 72, 21-27 (2015)

[5] Zagler et al., arXiv:1909.06372 (2019) 